\title{
Prosopagnosia following nonlanguage dominant inferior temporal lobe low-grade glioma resection in which the inferior longitudinal fasciculus was disrupted preoperatively: illustrative case
}

\author{
Jacob S. Young, MD, ${ }^{1}$ Ramin A. Morshed, MD, ${ }^{1}$ John P. Andrews, MD, ${ }^{1}$ Soonmee Cha, $M D,{ }^{2}$ and Mitchel S. Berger, $M{ }^{1}$
}

Departments of ${ }^{1}$ Neurological Surgery and ${ }^{2}$ Radiology, University of California, San Francisco, California

BACKGROUND Prosopagnosia is a rare neurological condition characterized by the impairment of face perception with preserved visual processing and cognitive functioning and is associated with injury to the fusiform gyrus and inferior longitudinal fasciculus (ILF). Reports of this clinical impairment following resection of right temporal lobe diffuse gliomas in the absence of contralateral injury are exceedingly scarce and not expected as a complication of surgery.

OBSERVATIONS The authors describe the case of a young female patient found to have an incidental diffuse glioma in the right inferior temporal lobe despite evidence of preoperative ILF disruption by the tumor. Following resection of the lesion, despite the preoperative disruption to the ILF by the tumor, the patient developed prosopagnosia. There was no evidence of contralateral, left-sided ILF injury.

LESSONS Given the significant functional impairment associated with prosopagnosia, neurosurgeons should be aware of the exceedingly rare possibility of a visual-processing deficit following unilateral and, in this case, right-sided inferior temporal lobe glioma resections. More investigation is needed to determine whether preoperative testing can determine dominance of facial-processing networks for patients with lesions in the right inferior posterior temporooccipital lobe and whether intraoperative mapping could help prevent this complication.

https://thejns.org/doi/abs/10.3171/CASE21277

KEYWORDS prosopagnosia; glioma; diffusion tensor imaging; temporal lobe; inferior longitudinal fasciculus

Social interactions rely on our ability to recognize faces and facial expressions. ${ }^{1}$ Electrophysiology, functional imaging, and direct cortical stimulation studies have provided evidence that the fusiform gyrus, basal occipital-temporal junction, and inferior longitudinal fasciculus (ILF) may play a special role in higher level visual processing and facial recognition. ${ }^{2,3}$ When familiar faces become unrecognizable despite the ability to identify facial features, such as the eyes, nose, and mouth, the condition is called prosopagnosia. Patients with prosopagnosia cannot identify a specific face, recognize whom the face belongs to, and do not know whether they ever saw the face before; the condition reached cultural significance in Oliver Sacks's book The Man Who Mistook His Wife for a Hat. Initially, bilateral insults to the fusiform gyri appeared to be necessary for patients to develop prosopagnosia, although, recently, it has been recognized that transient and/or permanent prosopagnosia may occur after unilateral right inferior posterior temporooccipital lesions or resections, although this appears to be exceedingly unlikely. $^{4-6}$

Still, despite these rare case reports of prosopagnosia following unilateral lesions or strokes, reports of this condition occurring after resection of a temporal lobe glioma are extremely sparse and effectively nonexistent in the glioma literature in the absence of bilateral injury, and this is not included in the preoperative conversation with the patient regarding the possible complications from surgery. Moreover, there is no consensus opinion in the neurological community that potential development of prosopagnosia can occur following

ABBREVIATIONS DTI = diffusion tensor imaging; IFOF = inferior frontal occipital fasciculus; ILF = inferior longitudinal fasciculus; LGG = low-grade glioma; $\mathrm{MRI}=$ magnetic resonance imaging.

INCLUDE WHEN CITING Published September 6, 2021; DOI: 10.3171/CASE21277.

SUBMITTED May 5, 2021. ACCEPTED May 24, 2021.

(c) 2021 The authors, CC BY-NC-ND 4.0 (http://creativecommons.org/licenses/by-nc-nd/4.0/). 

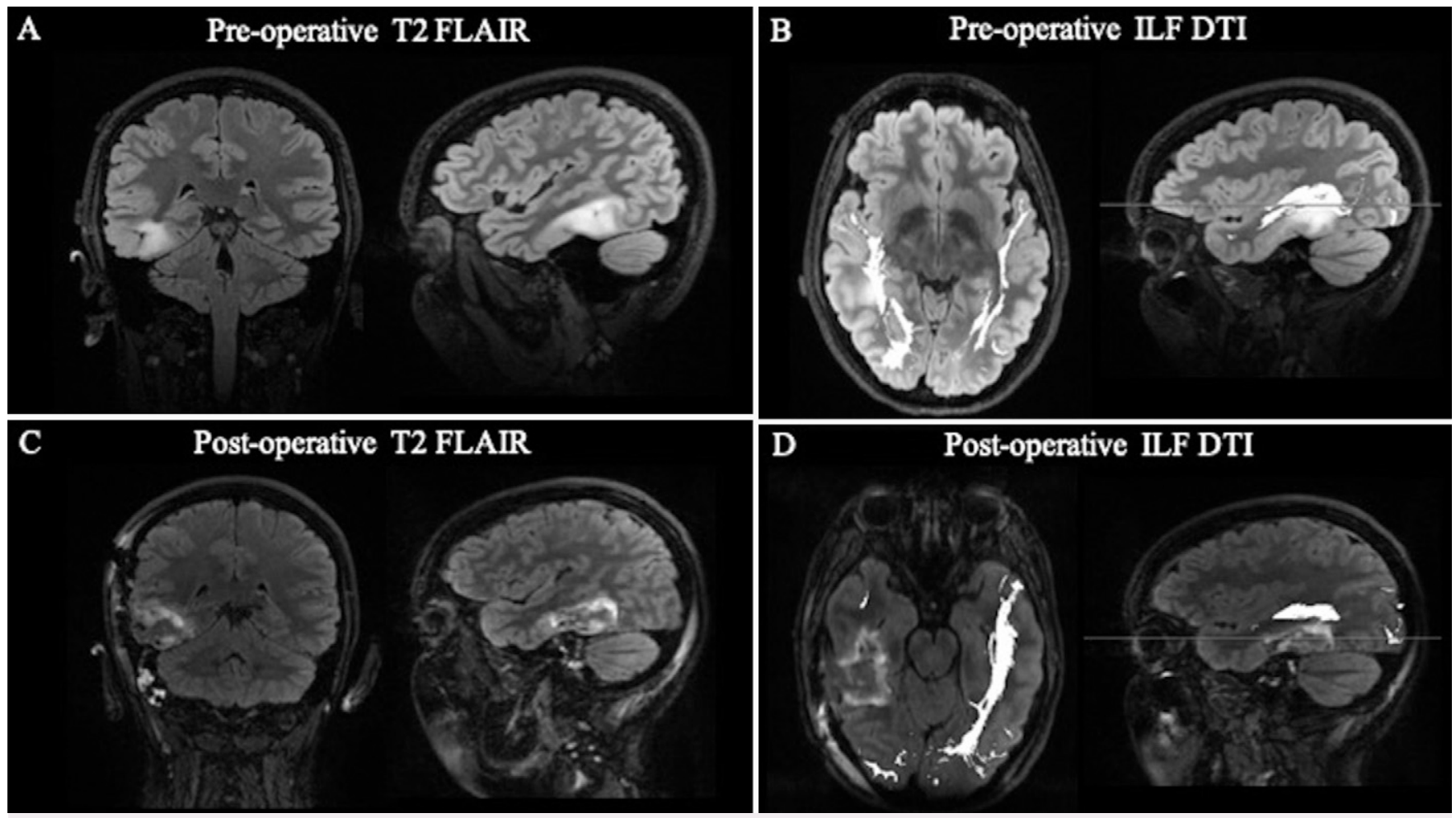

FIG. 1. A: Preoperative films demonstrating a T2 hyperintense lesion in the posterior inferior temporal gyrus and fusiform gyrus. B: Preoperative DTI showing disruption of the right ILF preoperatively but residual fibers are still present in the white matter fasciculus. C: Resection cavity is present within the right inferior temporal gyrus with small volume blood and a small region of cytotoxic edema along the superior medial margin of the resection cavity. D: Postoperative DTI showing additional transection of the ILF in the inferior temporal gyrus and fusiform gyrus. FLAIR = fluid-attenuated inversion recovery.

"nondominant," right hemisphere resections. This case illustrates a right temporal lesion where resection resulted in a postoperative prosopagnosia, and we discuss the relevant imaging characteristics and management considerations for lesions in this location.

\section{Illustrative Case}

This case describes a 22-year-old female patient who was found to have a T2 hyperintense, nonenhancing lesion in the right inferior temporal lobe, specifically within the cortical and subcortical inferior temporal gyrus and fusiform gyrus, which was concerning for a lowgrade glioma (LGG) during work-up for headaches associated with vertigo and nausea (Fig. 1A). The preoperative diffusion tensor imaging showed the presumed LGG was disrupting the fibers of the right ILF (Fig. 1B). She previously underwent a right stereotactic needle biopsy at an outside institution, and pathology revealed a diffuse astrocytoma, isocitrate dehydrogenase mutant. No neurological deficits were present on preoperative examination. Given the data supporting resection of incidentally identified low grade or diffuse gliomas, the patient underwent an asleep craniotomy for tumor resection. Postoperative magnetic resonance imaging (MRI) demonstrated that successful gross total resection was achieved, and postoperative diffusion tensor imaging (DTI) showed some mild change in the residual ILF (Fig. $1 \mathrm{C}$ and D). Minimal diffusion restriction was noted around the resection cavity (Fig. 2), as expected to occur following surgical resection.

Immediately postoperatively, the patient had a Karnofsky Performance Status of 80 . Unsurprisingly, there was a left upper quadrantanopia. Given that the lesion was in the nondominant hemisphere, her language comprehension and production were intact, as expected. However, the patient was completely unable to recognize faces. When other elements were added to the face, such as voice or characteristic clothing, she was able to correctly identify whom the face belonged to. The patient's postoperative prosopagnosia has persisted 5 months since surgery at the time of this publication.

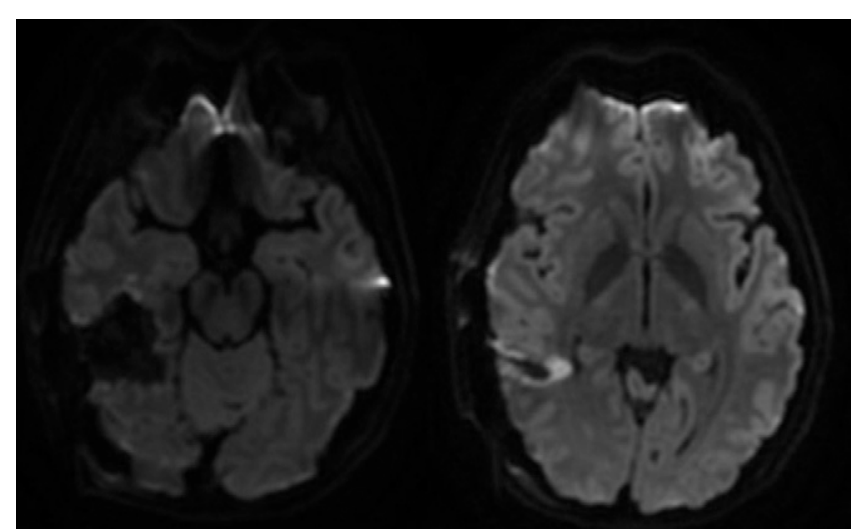

FIG. 2. Postoperative diffusion-weighted imaging demonstrating minimal reduced diffusion surrounding the resection cavity. 


\section{Discussion \\ Observations}

This case highlights a rare sequela following resection of a fusiform gyrus lesion in the nondominant language hemisphere. As shown in this case, preoperative DTI can be helpful to determine the spatial relationship between a lesion and ILF, but even when there is partial preoperative disruption of the ILF and an intact contralateral ILF, there could be a risk of a postoperative deficit in facial processing. While a few case reports have described similar prosopagnosia following resection of lesions in the right inferior posterior temporal-anterior occipital region, these reports often describe transient symptoms. ${ }^{4}$ Although early autopsy studies implied that bilateral injury was necessary to produce prosopagnosia, recent functional imaging studies and lesional case reports have suggested that right unilateral injury alone may be sufficient to produce functional impairment in face recognition; however, this has not been reported following unilateral tumor resection. ${ }^{7}$

Successful face recognition requires two connected cognitive processes: perception of face visual stimuli and matching this percept to face memory. The specific brain region usually associated with prosopagnosia is the fusiform gyrus, which has been shown to increase its activation specifically in response to faces. As mentioned above, some evidence suggests right hemisphere dominance regarding facial recognition processing;:-10 however, it frequently appears that bihemispheric interruptions are necessary for prosopagnosia to develop. ${ }^{11,12}$ Nevertheless, imaging studies have implied that the right hemisphere fusiform gyrus is more often involved in familiar face recognition than the left. ${ }^{13}$ For example, one study using transcranial magnetic stimulation to disrupt facial recognition found that facial-task accuracy was significantly impaired only during right and not left hemisphere stimulation. ${ }^{10}$ Anatomical studies of patients with congenital prosopagnosia have revealed reduced cortical volume of the fusiform gyrus ${ }^{14}$ and bihemispheric reductions in the integrity of the ILF and the inferior frontal occipital fasciculus (IFOF) white matter tracts. ${ }^{8,15}$ However, the degree of reduction in ILF and IFOF fibers only correlated with facial recognition impairment in the right hemisphere comparisons but not left hemisphere. $^{8}$ Variations in individual connectivity possibly explain why the etiology of this condition sometimes requires right-sided injury only and other times requires bilateral injury. ${ }^{9}$

The two cognitive processes of face perception and matching of the face perception to memory have led to two subtypes of prosopagnosia: apperceptive and associative. Apperceptive prosopagnosia describes cases of prosopagnosia associated with injury to processes early in the face perception system such as the fusiform gyrus, thus preventing identification of face stimuli., ${ }^{9,16}$ The brain areas thought to play a critical role in apperceptive prosopagnosia are right occipital temporal regions. People with this disorder cannot make any recognition of faces and are unable to make same-different judgments when they are presented with pictures of different faces. Comparatively, associative prosopagnosia has typically been used to describe cases of prosopagnosia where damage to the parahippocampal gyrus results in spared perceptual processes but impaired links between early face perception processes and the semantic memory information, preventing recognition of a face with the appropriate memories. Right anterior temporal regions appear to be involved in this process. People with this form of the disorder may be able to tell whether photographs of people's faces are the same or different and derive the age and sex from a face (suggesting they can make sense of some face information) but may not be able to subsequently identify the person or provide any information about them, such as their name or when they were last encountered.

In this report, we describe a patient who developed prosopagnosia after resection of the posterior fusiform gyrus/inferior temporal gyrus with diffusion-tensor imaging revealing a slight change in the residual ILF. The right ILF connects the anterior temporal pole with the fusiform face area and occipital cortex, which is sometimes referred to as the occipital face area, and has been demonstrated to be critical for high-level visual processing., ${ }^{1,17}$ Direct cortical stimulation of this white matter pathway can elicit a visual agnosia. ${ }^{2}$ Despite recognition of this condition for over 100 years, prosopagnosia is rarely reported as a deficit in the neurosurgical oncology literature, and, realizing this uncertainty, we were motivated to discuss our case. It should also be noted that the senior author of this report has never seen prosopagnosia in a postoperative patient following resections in the right or left temporal region in nearly 4000 glioma operations over his career before this case.

\section{Lessons}

This case demonstrates in rare cases the eloquence of the right posterior inferior temporal-occipital lobe and the ramifications that its resection can have on facial processing and the patient's quality of life. According to the dual-stream theory of visual processing, the ventral temporooccipital visual stream contains specialized regions for recognition of objects and, specifically, faces. ${ }^{18}$ Although prosopagnosia is exceedingly uncommon and appears to be rarely seen following right fusiform gyrus or ILF resection, this case highlights that the condition can occur following glioma resection and may warrant discussion with patients prior to resection.

It remains to be seen whether preoperative mapping of the "dominance" of the facial-processing system and/or whether awake resection with intraoperative visual testing during cases where the dominant, right posterior ILF is thought to be at risk can minimize the risk of this deficit. One potential method for preoperative identification of the face-processing network is repetitive transcranial magnetic stimulation, which has been shown to impair face processing when applied to the right occipital-face area but not the left occipital face area. ${ }^{10}$ Another strategy is to utilize functional MRI to locate regions most active in the face perception network. ${ }^{19}$

Moreover, it certainly appears that where the injury occurs along the anterior-posterior course of the ILF is relevant to the functional implications of ILF damage, as anterior temporal lobectomy invariably removes the anterior portion of the ILF and typically does not cause visual-processing disturbances. Interestingly, in the case presented, the ILF was already partially disrupted preoperatively by the tumor according to the tractography, suggesting that additional network disruption during surgery can lead to a deficit in the facialprocessing network despite an intact contralateral ILF.

While there is a preponderance of evidence supporting the use for awake-mapping approaches to identify and preserve motor and language functions during the resection of intraaxial lesions, fewer studies have investigated the use of awake-mapping techniques for the preservation of visual function (Table 1). ${ }^{20}$ Perhaps the lack of mapping studies for visual-processing lesions is the result of many patients presenting with preoperative quadrantanopia and the minimal impairment caused by quadrant defects in the visual field (in most states, patients can still drive following quadrant defects). Both cortical and subcortical mapping have been performed for occipital cortex lesions, and positive intraoperative identification of 
TABLE 1. Reports describing awake mapping of visual function

\begin{tabular}{lccc}
\hline \multicolumn{1}{c}{ Authors \& Year } & Task & $\begin{array}{c}\text { No. of } \\
\text { Patients }\end{array}$ & Findings \\
\hline Gras-Combe et al., $2012^{21}$ & 4 screen naming task & 14 & $\begin{array}{c}\text { Positive visual symptoms identified; } 1 \text { patient with } \\
\text { postoperative hemianopia; otherwise all patients } \\
\text { with expected quadrantanopia }\end{array}$ \\
\hline Duffau et al., $2004^{22}$ & No specific task & 1 & "Shadow" and "distortion" of visual field \\
\hline Coello et al., $2013^{2}$ & Naming pictures in opposite quadrants & 1 & $\begin{array}{c}\text { Inability to recognize picture in the contralateral } \\
\text { visual field }\end{array}$ \\
\hline Steno et al., $2012^{23}$ & $\begin{array}{c}\text { Fixed image displayed across all } 4 \text { quadrants } \\
\text { of the visual field and laser pointer to probe } \\
\text { different visual fields }\end{array}$ & 1 & Bright phosphenes in the visual field \\
\hline Nguyen et al., $2011^{24}$ & Red and green targets distributed in both \\
& visual fields & 1 & Flashing lights in the visual field
\end{tabular}

The table provides a list of prior publications investigating awake mapping of visual functions.

visual function can be reliably identified, either by producing a sensation of flashing lights in the patient's visual field or by generating transient vision loss. However, nearly all patients in these studies still develop a postoperative quadrantanopia, perhaps because this deficit can go unnoticed by the patient during the mapping. In these small studies exploring awake mapping, complete hemianopia was largely avoided, suggesting that visual deficits may be minimized with these awake-mapping approaches. Although the utility of mapping the visual system to preserve visual fields is debatable, mapping to prevent visual agnosia is even less researched. To our knowledge, there have not been any reports of mapping to prevent prosopagnosia during right-sided fusiform and ILF resections; however, Coello et al. report using a picture-recognition task with the screen displaying images in both hemifields to localize the right ILF near the fusiform gyrus. ${ }^{2}$

This case demonstrates a higher-order visual function that, to date, awake mapping has not routinely been utilized to preserve. Future work is needed to explore how intraoperative assessment of this higher-order sensory function as well as other cognitive and executive functions can be employed to optimize patient outcomes. Additionally, the dogma that the left hemisphere is the "dominant" hemisphere for all higher-order functions is challenged by this case. This case highlights the importance of nonmotor, nonspeech neurological functions in the "nondominant" right hemisphere, which are rarely discussed in the neurosurgical literature but still relevant for patient function and quality of life. In the future, tasks that identify eloquent regions for other nonlanguage, nonmotor cognitive processes may become more commonplace. Future investigation into the utility of awake mapping for facial-processing function is necessary to determine whether these rare neurological deficits can be minimized. Despite this being the only instance of prosopagnosia in the senior author's experience, neurosurgeons should be aware of the very rare possibility of a visual-processing deficit for right temporal lobe lesions.

\section{Acknowledgments}

We wish to acknowledge Katrina Scotford for helping us to understand the many facets of this unusual entity described in this report.

\section{References}

1. Fox CJ, laria G, Barton JJ. Disconnection in prosopagnosia and face processing. Cortex. 2008;44(8):996-1009.

2. Fernández Coello A, Moritz-Gasser S, Martino J, Martinoni M, Matsuda R, Duffau H. Selection of intraoperative tasks for awake mapping based on relationships between tumor location and functional networks. J Neurosurg. 2013;119(6):1380-1394.

3. Mandonnet E, Gatignol P, Duffau H. Evidence for an occipito-temporal tract underlying visual recognition in picture naming. Clin Neurol Neurosurg. 2009;111(7):601-605.

4. Mesad S, Laff R, Devinsky O. Transient postoperative prosopagnosia. Epilepsy Behav. 2003;4(5):567-570.

5. Wada $Y$, Yamamoto T. Selective impairment of facial recognition due to a haematoma restricted to the right fusiform and lateral occipital region. $J$ Neurol Neurosurg Psychiatry. 2001;71(2):254-257.

6. De Renzi E, Perani D, Carlesimo GA, Silveri MC, Fazio F. Prosopagnosia can be associated with damage confined to the right hemisphere-an MRI and PET study and a review of the literature. Neuropsychologia. 1994;32(8):893-902.

7. Landis T, Cummings JL, Christen L, Bogen JE, Imhof HG. Are unilateral right posterior cerebral lesions sufficient to cause prosopagnosia? Clinical and radiological findings in six additional patients. Cortex. 1986;22(2):243-252.

8. Thomas C, Avidan G, Humphreys K, Jung KJ, Gao F, Behrmann M. Reduced structural connectivity in ventral visual cortex in congenital prosopagnosia. Nat Neurosci. 2009;12(1):29-31.

9. Saygin ZM, Osher DE, Koldewyn K, Reynolds G, Gabrieli JD, Saxe RR. Anatomical connectivity patterns predict face selectivity in the fusiform gyrus. Nat Neurosci. 2011;15(2):321-327.

10. Pitcher D, Walsh V, Yovel G, Duchaine B. TMS evidence for the involvement of the right occipital face area in early face processing. Curr Biol. 2007;17(18):1568-1573.

11. Damasio AR, Damasio H, Van Hoesen GW. Prosopagnosia: anatomic basis and behavioral mechanisms. Neurology. 1982;32(4): 331-341.

12. Corrivetti F, Herbet G, Moritz-Gasser S, Duffau H. Prosopagnosia induced by a left anterior temporal lobectomy following a right temporo-occipital resection in a multicentric diffuse low-grade glioma. World Neurosurg. 2017;97:756.e1-756.e5.

13. Gainotti G, Marra C. Differential contribution of right and left temporo-occipital and anterior temporal lesions to face recognition disorders. Front Hum Neurosci. 2011;5:55. 
14. Behrmann M, Avidan G, Gao F, Black S. Structural imaging reveals anatomical alterations in inferotemporal cortex in congenital prosopagnosia. Cereb Cortex. 2007;17(10):2354-2363.

15. Grossi $D$, Soricelli A, Ponari $M$, et al. Structural connectivity in a single case of progressive prosopagnosia: the role of the right inferior longitudinal fasciculus. Cortex. 2014;56:111-120.

16. Kanwisher N, McDermott J, Chun MM. The fusiform face area: a module in human extrastriate cortex specialized for face perception. J Neurosci. 1997;17(11):4302-4311.

17. Cohen AL, Soussand L, Corrow SL, Martinaud O, Barton JJS, Fox MD. Looking beyond the face area: lesion network mapping of prosopagnosia. Brain. 2019;142(12):3975-3990.

18. Barton JJ. Disorder of higher visual function. Curr Opin Neurol. 2011;24(1):1-5.

19. Pyles JA, Verstynen TD, Schneider W, Tarr MJ. Explicating the face perception network with white matter connectivity. PLoS One. 2013;8(4):e61611.

20. Wolfson $\mathrm{R}$, Soni $\mathrm{N}$, Shah $\mathrm{AH}$, et al. The role of awake craniotomy in reducing intraoperative visual field deficits during tumor surgery. Asian J Neurosurg. 2015;10(3):139-144.

21. Chan-Seng E, Moritz-Gasser S, Duffau H. Awake mapping for lowgrade gliomas involving the left sagittal stratum: anatomofunctional and surgical considerations. J Neurosurg. 2014;120(5):1069-1077.

22. Duffau H, Velut S, Mitchell MC, Gatignol P, Capelle L. Intra-operative mapping of the subcortical visual pathways using direct electrical stimulations. Acta Neurochir (Wien). 2004;146(3):265-270.
23. Šteňo A, Karlík M, Mendel P, Čík M, Šteňo J. Navigated threedimensional intraoperative ultrasound-guided awake resection of low-grade glioma partially infiltrating optic radiation. Acta Neurochir (Wien). 2012;154(7):1255-1262.

24. Nguyen HS, Sundaram SV, Mosier KM, Cohen-Gadol AA. A method to map the visual cortex during an awake craniotomy. J Neurosurg. 2011;114(4):922-926.

\section{Disclosures}

The authors report no conflict of interest concerning the materials or methods used in this study or the findings specified in this paper.

\section{Author Contributions}

Conception and design: Berger, Young. Acquisition of data: Berger, Young. Analysis and interpretation of data: all authors. Drafting the article: all authors. Critically revising the article: Berger, Young, Morshed, Andrews. Reviewed submitted version of manuscript: Berger, Young, Morshed, Andrews. Approved the final version of the manuscript on behalf of all authors: Berger. Statistical analysis: Berger. Administrative/technical/material support: Berger, Cha. Study supervision: Berger.

\section{Correspondence}

Mitchel S. Berger: University of California, San Francisco, CA. mitchel.berger@ucsf.edu. 